\title{
A steady course towards the next tokamak
}

\section{Tokyo}

RESEARCHERS at the Japan Atomic Energy Research Institute (JAERI) north of Tokyo have begun to design a steady-state tokamak to succeed the institute's aging JT-60 tokamak. Proponents argue that it is needed to preserve Japan's role in developing fusion devices for the next century, but critics question whether it is the best use of scarce resources.

Fuelled with money appropriated for the fiscal year that began last week, JAERI researchers will join their industrial counterparts at Hitachi, Toshiba and Mitsubishi in completing the design of a steady-state tokamak that is intended to be a stepping stone between JT-60 and the International Thermonuclear Experimental Reactor (ITER). The \$6,000-million ITER project is expected to be a combined effort of the United States, Japan, Europe and the Soviet Union.

The aim is to make a device that can maintain a heated deuterium-deuterium plasma for 100 to 1,000 seconds, much longer than the short bursts of a few seconds that current tokamaks like JT-60 achieve. And it would be a final step before making a device such as ITER that can exceed the 'break-even' point and produce more energy than it consumes.

The JAERI initiative, which has yet to win government approval, matches a similar move to build a steady-state device in the United States (see Nature 356, 96; 1992). It is motivated partly by fear that the institute's scientists will be left without an advanced fusion device of their own when JT-60 reaches the end of its life.

Just as the US proposal for a Tokamak Physics Experiment came after fusion scientists failed to win funding for the more ambitious and more costly Burning Plasma
Experiment, the JAERI steady-state plan comes after the institute's plan to build a Fusion Experimental Reactor was shelved by Japan's Atomic Energy Commission. The commission instead cast its lot with an effort that would lead directly to the ITER prototype reactor.

US scientists are driven by the knowledge that the country's leading

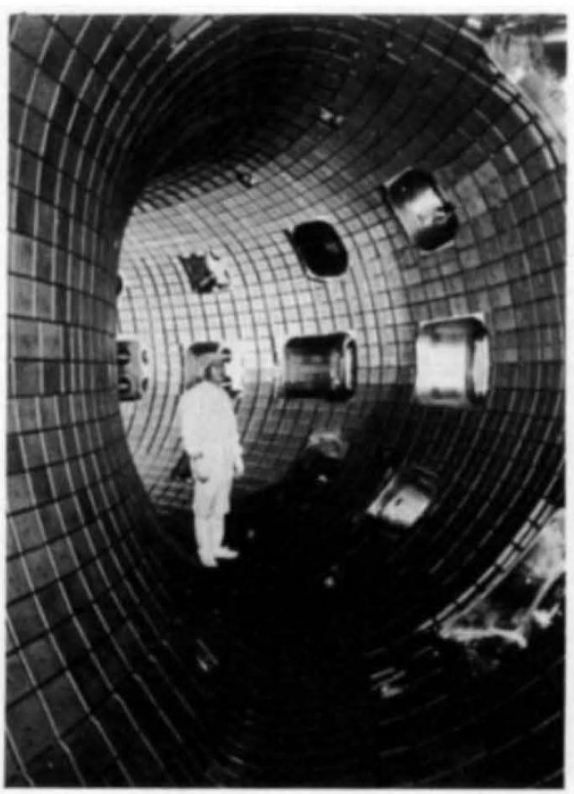

A worker stands inside the JT-60 machine during its recently completed renovation.

tokamak, in Princeton, will reach the end of its scientific life in 1995. But the Japanese face a less urgent situation. The JT60 , which has just undergone a major renovation, is thought to be good for another dozen or so years.

Although the planned steady-state device will use the existing power facilities

\section{Helical fusion machine on course}

JAPAN's plans to build the world's largest helical fusion device are proceeding "almost on schedule", says Ikuo liyoshi, director of Japan's new National Institute for Fusion Science. The device, which is under construction at Toki in Gifu Prefecture near Nagoya (see Nature 335,$104 ; 1988$ ), represents a key part of Japan's fusion research that is funded by the Ministry of Education, Science and Culture. In contrast, research at the Japan Atomic Energy Research Institute (JAERI) is funded by the Science and Technology Agency.

The new institute will have a staff of 280 , including 150 researchers, when it opens in 1995. It is based temporarily at Nagoya University. The device will cost $¥ 50,000$ million (US $\$ 370$ million), but only about one-tenth of that amount has been spent so far.

This year, some $¥ 4,500$ million (US $\$ 33$ million) will be spent on the fabrication of machines to make superconducting magnets, and a similar amount for new buildings. The institute also plans to buy a supercomputer, liyoshi says.

The helical device will produce a magnetic energy of 2 gigajouls and will be by far the largest such device in the world. Its purpose is to examine the physics of plasmas under steady-state conditions, a mission not linked directly to the development of commercial fusion reactors. for the JT-60, most of it will be built from scratch, including the vacuum vessel and magnets. Unlike the current tokamaks, the new device will use superconducting magnets and special robot equipment. The superconducting magnets generate stronger fields in a more compact area and at lower operating costs, and the robots are the only way to maintain the walls of the tokamak, which become radioactive after confining the deuterium-deuterium plasma for long periods of time.

JAERI researchers declined to comment on the expected cost of the new machine. But US scientists who saw preliminary design plans of the Japanese tokamak at a meeting last November estimate that the Japanese machine would cost about $\$ 1,000$ million, or double the price of the US machine. The US Burning Plasma Experiment would have cost $\$ 1,500$ million.

The Japanese machine is expected to take about four years to build. JAERI officials would like to be using it before the end of the decade, meaning that a formal proposal must be submitted to the Japanese government in the next few years if that timetable is to be met.

Despite all the planning that has taken place, some Japanese fusion scientists question whether this project should move forward. Atsuo Iiyoshi, director of Japan's new National Institute for Fusion Science, says "I don't disagree with their plan". But if fusion budgets are going to be limited, he says, it would be better "to divide roles" among existing programmes.

One such effort is a giant helical fusion device under construction at liyoshi's institute. Its prime purpose is to examine steady-state experiments in fusion (see below). Iiyoshi suggests that JAERI could conduct research involving burning plasmas by using tritium in the JT- 60 , while Iiyoshi's new fusion institute looks at the characteristics of steady-state experiments.

But JAERI researchers do not want to burn tritium in the JT- 60 . That is one of the research aims of scientists at Princeton and the JET (Joint European Torus) tokamak in Britain, they say.

The fate of the JAERI plan is uncertain. The timing of a proposal for a follow-up to JT-60 is "delicate", says Ikuo Makino, director of the Technology Development Division of the Atomic Energy Bureau at the Science and Technology Agency, which is in charge of JAERI. Such a project, he says, must not compete with the construction of ITER, which could begin in the latter half of the 1990 s for completion by 2005 . Still, it is clear that JAERI has made the steady-state machine a priority. 